\title{
High BTBD7 expression positive is correlated with SLUG-predicted poor prognosis in hormone receptor-negative breast cancer
}

\author{
Zi-Xiong Li ${ }^{1,2,3}$, Ze-Nan Huang ${ }^{4}$, Hui Luo ${ }^{5}$, Xiong-Bin Yang ${ }^{1}$, Yu-Lin Wang ${ }^{6}$, Jie-Xin Chen ${ }^{7}$, Xiao-Kai Ma $^{8}$, \\ Feng $\mathrm{Xu}^{2,9}$, Tian-Tian Wang ${ }^{10}$, Ling Lin ${ }^{1}$ \\ ${ }^{1}$ Department of Rheumatology, The First Affiliated Hospital, Shantou University Medical College, Shantou, China; ${ }^{2}$ Clinical Research Center, \\ The First Affiliated Hospital of Shantou University Medical College, Shantou, China; ${ }^{3}$ Guangdong Provincial Key Laboratory for Diagnosis and \\ Treatment of Breast Cancer, Shantou University Medical College, Shantou, China; ${ }^{4}$ Department of Thyroid and Breast Surgery, The Third Affiliated \\ Hospital of Sun Yat-sen University, Guangzhou, China; ${ }^{5}$ Anesthesia and Operation Center, The Third Affiliated Hospital of Sun Yat-sen University, \\ Guangzhou, China; ${ }^{6}$ Department of Neurosurgery, First Affiliated Hospital, Shantou University Medical College, Shantou, China; ${ }^{7}$ Department \\ of Endocrinology, First Affiliated Hospital, Shantou University Medical College, Shantou, China; ${ }^{8}$ The first affiliated Hospital of Bengbu Medical \\ College, Bengbu, China; ${ }^{9}$ Department of Respiratory and Critical Care Medicine, The First Affiliated Hospital of Shantou University Medical \\ College, Shantou, Guangdong, China; ${ }^{10}$ Department of Medical Oncology, The Third Affiliated Hospital, Sun Yat-sen University, Guangzhou, \\ China \\ Contributions: (I) Conception and design: TT Wang, ZX Li, L Lin; (II) Administrative support: None; (III) Provision of study materials or patients: \\ ZX Li, ZN Huang, H Luo, XB Yang, JX Chen; (IV) Collection and assembly of data: XB Yang, F Xu, ZX Li; (V) Data analysis and interpretation: \\ YL Wang, ZX Li, XK Ma; (VI) Manuscript writing: All authors; (VII) Final approval of manuscript: All authors. \\ Correspondence to: Tian-Tian Wang. Department of Medical Oncology, The Third Affiliated Hospital, Sun Yat-sen University, Guangzhou, China. \\ Email: wangtt37@mail.sysu.edu.cn; Ling Lin. Department of Rheumatology, The First Affiliated Hospital, Shantou University Medical College, \\ Shantou, China. Email: 1lin6@stu.edu.cn.
}

Background: Hormone receptor-negative breast cancer (HRNBC), which includes triple-negative breast cancer (TNBC) and human epidermal growth factor receptor 2 (HER-2) overexpressing breast cancer, is prone to metastasis and has a poor prognosis. BTB/POZ domain-containing protein 7 (Btbd7) is thought to regulate SLUG and the epithelial-mesenchymal transition (EMT) process. However, the role of Btbd7 in HRNBC is unclear.

Methods: Expression of BTBD7 and SLUG in HRNBC tumor tissue and normal adjacent tissue (NAT) as well as breast cancer cells were characterized by immunohistochemistry and immunofluorescence. MDAMA-231 cells was transfected with BTBD7 siRNA and detected by qRT-PCR and western blot. Expression levels of Slug and EMT related proteins were detected western blot analysis. cell invasion assays were used to analyse cell invasion ability of MDA-MA-231. GO and KEGG analyses was used to analysis the gene function.

Results: The total positive rate of BTBD7 expression in HRNBC tumor tissue was 66.7\%, which was higher than that in NAT $(52.1 \%)$ and benign breast lesion tissues (20\%). Co-expression of SLUG and BTBD7 proteins could be found in HRNBC tissue and MDA-MA-231 cells. BTBD7 silencing significantly up-regulated the epithelial marker E-cadherin, down-regulated the mesenchymal markers $\alpha$-SMA and SLUG and suppressed the invasion abilities of MDA-MA-231 cells. GO and KEGG analyses based on 322 DEGs showed that BTBD7 may be associated with generic transcription in breast cancer.

Conclusions: The study data indicated that BTBD7 was inversely associated with SLUG expression. Higher BTBD7 was associated with poor clinicopathologic features and prognosis in HRNBC patients. $B T B D 7$ silencing inhibited EMT through regulation of SLUG expression. BTBD7 might act as a potential molecular target for gene therapy in HRNBC patients.

Keywords: BTB/POZ domain-containing protein 7 (BTBD7); SLUG; epithelial-mesenchymal transition (EMT); hormone receptor negative breast cancer (HRNBC) 
Submitted Jun 10, 2021. Accepted for publication Aug 05, 2021.

doi: 10.21037/atm-21-3409

View this article at: https://dx.doi.org/10.21037/atm-21-3409

\section{Introduction}

According to the International Agency for Research on Cancer, breast cancer is the most common malignant tumor and the leading cause of cancer-related deaths among women worldwide (1). Breast cancer is clinically categorized into four therapeutic groups according to its hormone receptor and HER-2 status. Luminal A and Luminal B are hormone receptor-positive breast cancer and both HER-2 overexpression and triple-negative breast cancer (TNBC) are hormone receptor-negative breast cancers (HRNBC) (2-4).

HER-2 overexpression is defined as a lack of the expression of hormonal receptor (HR) and overexpression of HER-2 in breast cancer cells, and accounts for 10-25\% of the breast cancer cases (5). While overexpression of the HER-2 gene is a poor prognostic factor, the development of HER-2 targeted therapy has revolutionized the treatment of these cancers. However, drug resistance to trastuzumab may lead to only transient benefits. TNBC is defined by the lack of expression of HR and HER-2 in breast cancer cells (6-8), and accounts for approximately $10 \%$ of all breast cancer cases worldwide (9). TNBC is characterized by a younger onset age, larger tumor size, and higher rate of lymphatic and distant metastases $(10,11)$. Existing therapeutic options for TNBC, are surgery, chemotherapy, and radiotherapy, which often have limited effects. Although HRNBC was more sensitive to chemotherapy than hormone receptorpositive breast cancer, because they are highly malignant and can't benefit from endocrine therapy, their prognosis will be worse.

EMT is generally accepted as a hallmark of tumor invasion and metastasis in breast cancer, and during this process, the polarity, adhesion ability, and differentiation characteristics of mesenchymal tissues are altered due to biochemical changes in normal mammary epithelial cells $(12,13)$. SLUG, also known as snail2, is known to be a regulatory factor for induction of the EMT process and acts by repressing E-cadherin gene transcription via E-box elements, specifically EboxA and EboxC (14-16).

BTB/POZ domain-containing protein 7 (BTBD7) has a molecular weight of $126 \mathrm{kD}$ and is a member of the brica-brac tramtrack broad complex (BTB) protein family. It has a conserved $\mathrm{BTB} / \mathrm{POZ}$ protein-protein interaction motif (17), which is identified as a critical regulatory factor in epithelial cell dynamics and branching morphology (18). Research on embryonic development found that high focal expression of BTBD7 maybe induced by fibronectin or other matrix proteins, and led to the local regulation of SLUG, E-cadherin, and epithelial cell motility (19). BTBD7 has been proven to promote metastasis and be an adverse prognostic factor in non-small cell lung cancer (NSCLC) (20), hepatocellular carcinoma (21), and human salivary adenoid cystic carcinoma (SACC) (22), and RhoCRock2-FAK-signaling pathway maybe the regulation of BTBD7. However, whether BTBD7 participates in the development of metastasis and affects the prognosis of HRNBC is unclear. In this study, we aimed to investigate the role of BTBD7 in HRNBC prognosis. To this end, we analysed $B T B D 7$ expression in breast cancer using The Cancer Genome Atlas (TCGA), immunohistochemistry (IHC), and conducted in vitro experiments to investigate the localization and function of BTBD7 in cancer cells.

We present the following article in accordance with the REMARK reporting checklist (available at https://dx.doi. org/10.21037/atm-21-3409).

\section{Methods}

\section{Tissue samples}

This study was performed on 30 paraffin-embedded tissues isolated from benign breast lesions (15 were identified as fibrocystic mastopathy, while the other 15 were breast fibroadenoma), 144 paraffin-embedded tissues isolated from patients with HRNBC ( 94 were TNBC tissues, and 50 were HER-2 overexpressing breast cancer tissues), including tumor tissue and NAT, which were selected $3-5 \mathrm{~cm}$ away from the site at which the primary tumor was obtained. All materials were obtained from The Third Affiliated Hospital of Sun Yat-sen University and The First Affiliated Hospital, Shantou University Medical College, between 2007 and 2016. The inclusion criteria were that all patients were treated surgically with radical mastectomy or conservative quadrantectomy in these two hospitals; all underwent postoperative chemotherapy or radiation therapy conforming to these guidelines; the pathological pattern was invasive ductal carcinoma (IDC); and estrogen 
receptor (ER) and progesterone receptor (PR) were negative. Clinicopathological data, including age, sex, type of surgery, HER-2 receptor, tumor size, location, tumor node metastasis (TNM) staging, histology, and lymphatic invasion, were collected retrospectively from the inpatient records and pathology department. All patients completed a telephone follow-up interview after the initial surgery. Patients excluded from the study were those who had additional cancers or other life-threatening diseases, or had distant metastasis before the operation.

TNBC and HER-2 overexpression breast cancer patients were categorized according to the tumor-node-metastasis staging system classification (American Joint Committee on Cancer, AJCC). Tumor cells exhibiting greater than or equal to $10 \%$ positivity for ER or PR at any staining intensity were considered hormone receptor-positive. The HER-2 staining score was evaluated from 0 to $3+$. A HER-2 score of $0-1+$ was considered negative, and when the HER-2 score was $2+$ and $3+$, further examination of fluorescence in situ hybridization (FISH) was performed (Figure S1).

Patient follow-up was terminated on August 26, 2018. The age range of patients was 24 to 86 years at the time of surgery, with an average age of 50.4 years, and the followup period ranged from 27 to 130 months, with an average of 75 months. Disease free survival (DFS) was calculated from the time of breast cancer resection to the first radiological evidence of recurrence or the last observation, and overall survival (OS) as the time interval between breast cancer resection and death or the last observation.

Ethics approval was obtained from the ethics committee of The Third Affiliated Hospital of Sun Yat - sen University (No. [2018]-081) and the First Affiliated Hospital, Shantou University Medical College (No. [2018]-027). Samples were anonymously coded in accordance with local ethical guidelines [as stipulated by the Declaration of Helsinki (as revised in 2013)]. Written informed consent was obtained from study participants.

\section{Immunobistochemical staining and scoring for BTBD7 and SLUG expression}

Each tissue section $(5 \mu \mathrm{m} \times 5 \mu \mathrm{m})$ was dewaxed, rehydrated, and treated with $0.3 \%$ hydrogen peroxide to block endogenous peroxidase, followed by antigen retrieval. The sections were incubated with rabbit anti-BTBD7 (1:100, ab204362; Abcam) and mouse anti-SLUG (1:20, sc-166476, Santa Cruz Biotechnology) overnight at $4{ }^{\circ}$ C. After washing, the bound antibodies were detected using horseradish peroxidase-conjugated secondary antibody (Maxin, Fujian, China) and diaminobenzidine (Xilong Scientific, China), followed by counterstaining with hematoxylin (Keygen Biotech, Nanjing, China). The primary antibody was omitted from the negative control samples.

The evaluation and scoring of BTBD7 and SLUG expression were conducted by two independent investigators with pathological training following a blind protocol. The IHC results of BTBD7 and SLUG staining were evaluated by multiplying the scores of proportions of positively stained cells by their staining intensity scores. For BTBD7 and SLUG, the staining intensity was visually scored as 0 (no staining at all), 1 (weak), 2 (medium), or 3 (strong). The staining extent was also scored as $0(0-10 \%), 1(10-24 \%), 2$ (25-50\%), 3 (51-75\%), or 4 ( $\geq 75 \%)$. A multiplicative score of 2 or more was considered as a positive staining.

\section{Cell lines and cell culture}

Human breast cancer cells MDA-MB-231, SKBR-3, and MCF-10A were obtained from the American Type Culture Collection (ATCC) and were cultured at $37{ }^{\circ} \mathrm{C}$ and $5 \% \mathrm{CO}_{2}$ in DMEM (high glucose) supplemented with $10 \%$ fetal bovine serum.

\section{Reverse transcription-quantitative polymerase chain reaction}

The forward primer for BTBD7 was 5'-AAAGGAGCTTT CTCTACAAGCC-3' and the reverse primer was 5'-GCC CCATACTCTGGTGAGGAA-3'. Relative mRNA expression levels were calculated using the $2^{-\Delta \mathrm{Ct}}$ method, based on threshold cycle values, and were normalized to the internal control.

\section{siRNA transfections}

Silencer Select siRNA targeting BTBD7 was obtained from GenePharma Shanghai. The siRNA1 sequence was CAAGTATTGTACTGATGTA, and the siRNA2 sequence was CCCGGACATTGCAGAAAGA. For transient transfection, the cells were cultured in a 24well plate for $24 \mathrm{~h}$ before the experiment, then transfected with Lipofectamine 2000 according to the manufacturer's instructions. Following transfection, the cells were harvested at $24-48 \mathrm{~h}$ to measure protein and gene expression levels. 


\section{Western blot analysis}

Total protein was separated by sodium dodecyl sulfate polyacrylamide gel electrophoresis and then transferred to polyvinylidene fluoride membranes. Blocked membranes were incubated with the primary antibodies, including BTBD7 (1:1,000 ab204362, Abcam), SLUG (1:100, sc166476, Santa Cruz Biotechnology), E-cadherin (1:2,000 ab40772, Abcam), and $\alpha$-SMA (1:1,000, 19245, Cell Signaling Technology), followed by horseradish peroxidaseconjugated secondary antibodies (AS005 Asbio). Finally, protein expression was examined using an ECL kit. Densitometry measurements were performed using ImageJ software.

\section{Matrigel invasion assay}

The invasive abilities of the cells were examined using a 24well transwell with 8 - $\mu \mathrm{m}$ pore polycarbonate membrane inserts (Corning, NY, USA) and Matrigel (BD Bioscience) according to the manufacturer's instructions. Matrigel $20 \mu \mathrm{L}$ (1:3 dilution) was added to each insert, and $100 \mu \mathrm{L}$ of cell suspension containing $3 \times 10^{5}$ cells were transferred to the upper chamber and incubated for 36-48 h. The filters were stained with hematoxylin, and cells that appeared on the lower surface of the filter were counted in five random high-magnification microscopes. Each experiment was performed three times independently.

\section{Immunofluorescent staining}

Cells were fixed with $4 \%$ paraformaldehyde, blocked with $1 \%$ BSA, and tissue sections were dewaxed and debenzolized. Cells were then incubated with rabbit antiBTBD7 (1:400 dilution, ab204362; Abcam, Cambridge, MA) and mouse anti-SLUG (1:100 dilution, sc-166476, Santa Cruz Biotechnology) antibodies overnight at $4{ }^{\circ} \mathrm{C}$. The secondary antibodies were goat anti-mouse Alexa Fluor 546 (Invitrogen A-11030) and goat anti-rabbit Alexa Fluor 488 (Invitrogen A-11008). Nuclei were counterstained with DAPI, and cells and tissue sections were examined using a Zeiss LSM880 confocal fluorescent microscope (Zeiss, Germany).

\section{Bioinformatics analysis}

Gene expression data of 1,082 breast cancer samples (23) were obtained from Affymetrix HGU133A and HGU133+2 microarrays and divided into $B T B D 7$ low- and highexpression groups according to the median expression value of BTBD7. R package 'edgeR' ( $\mathrm{R}$ version 3.5.2) was used to identify DEGs, and genes with correlation coefficients greater than 0.55 or less than -0.55 and false discovery rate $(\mathrm{FDR})<0.05$ were considered as DEGs. To explore the functional roles of $B T B D 7$, GO was performed on the DEGs using DAVID (https://david.ncifcrf.gov/) (24). Terms with FDR $<0.05$ were selected and visualized using R language, and KOBAS 3.0 (http://kobas.cbi.pku.edu.cn/ kobas3/?t=1) (25) was used to confirm the GO and KEGG terms in DAVID. GO terms with corrected $\mathrm{P}<0.05$, and terms of the KEGG pathway with $\mathrm{P}<0.05$, are listed.

\section{Statistical analysis}

SPSS for Windows version 21.0 was used for data analyses. All experiments were performed in triplicate, and the data were expressed as means \pm SD and analysed using Student's $t$-test. For overall survival OS and DFS, the Kaplan-Meier method was used. The un-paired $t$-test was performed to identify the DEGs, and the paired $t$-test was used to compare the IHC finding of protein expression between NAT and tumor tissue. Univariate and multivariate Cox regression models were used to determine the relationship between multiple variables and OS and DFS. A P value of $<0.05$ was considered statistically significant.

\section{Results}

\section{Demographic and clinicopathological characteristics of HRNBC patients}

Overall, 144 HRNBC patients were studied, and their demographic and clinicopathological characteristics are shown in Table 1. All patients were women, and 76 (52.8\%) were younger than 50 , while $68(47.2 \%)$ were 50 or older. A total of 113 (78.5\%) and 31 (21.5\%) patients had tumors with TNM stages I-II and III, respectively, and thirty (20.8\%) and 114 (79.2\%) patients had T classifications of T1 and T2-T4, respectively. In total, 57 (39.6\%) patients were devoid of lymph node invasion. Forty-eight (33.3\%) and 96 $(66.7 \%)$ patients had well + moderate cell differentiation and poor cell differentiation of IDC, respectively, while fifty (34.7\%) patients had HER-2 positivity. During the followup period, 29 (20.1\%), 19 (10.5\%), 10 (6.9\%), 16 (11.1\%), and $3(2.1 \%)$ patients had lung, bone, liver, chest wall, and brain metastasis, respectively, and 33 (22.9\%) patients died. 
Table 1 Demographic and clinicopathological characteristics of 144 hormone receptor negative breast cancer

\begin{tabular}{|c|c|c|}
\hline Variables & No. & $\%$ \\
\hline \multicolumn{3}{|l|}{ Age(years) } \\
\hline$<50$ & 76 & 52.8 \\
\hline$\geq 50$ & 68 & 47.2 \\
\hline \multicolumn{3}{|l|}{ TNM stage } \\
\hline I-II & 113 & 78.5 \\
\hline III & 31 & 21.5 \\
\hline \multicolumn{3}{|l|}{ T classification } \\
\hline $\mathrm{T} 1$ & 30 & 20.8 \\
\hline T2-4 & 114 & 79.2 \\
\hline \multicolumn{3}{|l|}{$\mathrm{pN}$} \\
\hline NO & 87 & 60.4 \\
\hline N1-3 & 57 & 39.6 \\
\hline \multicolumn{3}{|l|}{ IDC Grading } \\
\hline Well + moderate & 48 & 33.3 \\
\hline Poor & 96 & 66.7 \\
\hline \multicolumn{3}{|l|}{ HER-2 } \\
\hline Negative & 94 & 65.3 \\
\hline Positive & 50 & 34.7 \\
\hline \multicolumn{3}{|l|}{ Metastatic sites } \\
\hline Lung & 29 & 20.1 \\
\hline Bone & 19 & 13.2 \\
\hline Liver & 10 & 6.9 \\
\hline Chest wall & 16 & 11.1 \\
\hline Brain & 3 & 2.1 \\
\hline
\end{tabular}

\section{Expression of BTBD7 in HRNBC and benign breast lesions and its relationships with clinicopathological parameters}

IHC analysis was performed to investigate BTBD7 expression in HRNBC and benign breast lesion tissues. Positive immunohistochemical staining of BTBD7 was mainly observed in the cytoplasm of the cells, while benign breast lesions and NAT cells exhibited negative or weaker staining when compared with HRNBC cells (Figure 1A). The total positive rate of BTBD7 expression in HRNBC tumor tissue was $66.7 \%(96 / 144)$, which was higher than that in NAT $(52.1 \% 75 / 144, \mathrm{P}<0.001)$ and benign breast lesion tissues $(20 \%, 6 / 30)$ (Figure $1 B, \mathrm{P}<0.001)$. Increased BTBD7 expression in HRNBC was significantly associated with larger tumor volume and poorer TNM stage (Figure $1 B$, $\mathrm{P}<0.05)$. However, no significant association was observed between the expression of BTBD7 and other clinicopathological factors in HRNBC.

\section{Survival analysis}

The data from TCGA showed that in HRNBC patients and those in the HER-2 overexpression breast cancer subgroup, higher $B T B D 7 \mathrm{mRNA}$ expression tended to be associated with shorter DFS $(\mathrm{P}<0.001$, Figure $1 C, 1 D)$. However, the DFS in patients with TNBC was insufficient to adequately analyse prognosis. As shown by the Kaplan-Meier survival curves in Figure 1E, $1 F$, the median estimated DFS $(93.5 \pm 5.0$ vs. $72.7 \pm 5.8, \mathrm{P}=0.003)$ and $\mathrm{OS}(98.4 \pm 3.8$ vs. $92.2 \pm 5.2$, $\mathrm{P}=0.028)$ in patients with low BTBD7 expression were longer than in those with high BTBD7 expression.

To analyse the function of BTBD7 in TNBC and HER$2+$ patients, we performed a subgroup analysis of these two molecular subtypes.

In the TNBC subgroup analysis, patients with high BTBD7 expression had significantly shorter DFS $(\mathrm{P}=0.049)$ and $\mathrm{OS}(\mathrm{P}=0.048)$ than those with low BTBD7 expression (Figure $1 G, 1 H$ ). As shown in Figure S2A-J, among patients aged $<50$ years and with TNM stages $1-2$ and well + moderately differentiated IDC, a higher BTBD7 expression level was associated with shorter DFS, and as shown in Figure S2K-T, among patients aged $>50$ years and with lymph node metastasis, a higher BTBD7 expression level was associated with shorter OS time.

In the HER-2+ subgroup analysis, patients with high BTBD7 expression had significantly shorter DFS $(\mathrm{P}=0.026)$ than those with low BTBD7 expression ( $\mathrm{P}=0.026$, Figure 11). However, no significant differences were observed in the median estimated survival time between HER-2+ patients with low and high BTBD7 expression ( $\mathrm{P}=0.338$, Figure 17). As shown in Figure $\mathrm{S} 3 \mathrm{~A}-\mathrm{S} 3 \mathrm{~J}$, among patients aged $>50$ years, tumor diameter $>2 \mathrm{~cm}$, lymph node metastasis, and poorly differentiated IDC, a higher BTBD7 expression level was associated with shorter DFS time. However, as shown in Figure S3K-S3T, a higher BTBD7 expression level was not associated with shorter OS time among subgroups.

Tumor recurrence and metastasis are the main causes of death in breast cancer patients. We found that high BTBD7 expression was significantly associated with a higher lung metastasis rate $(28.1 \%, 27 / 96)$ in comparison to patients with low BTBD7 expression $(4.2 \%, 2 / 48, \mathrm{P}=0.001)$, 
A

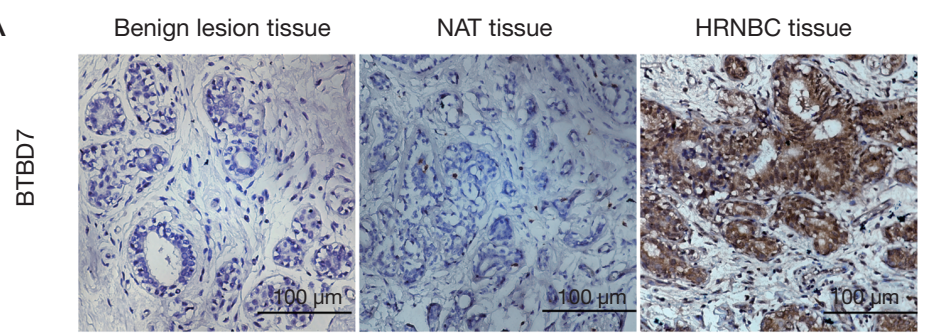

B

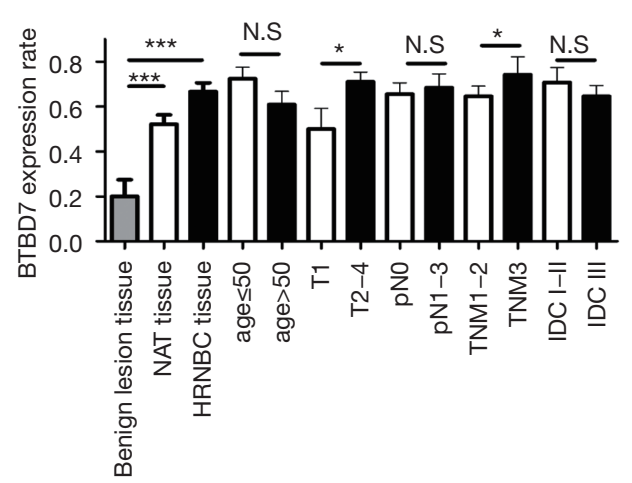

C

HRNBC (TCGA)

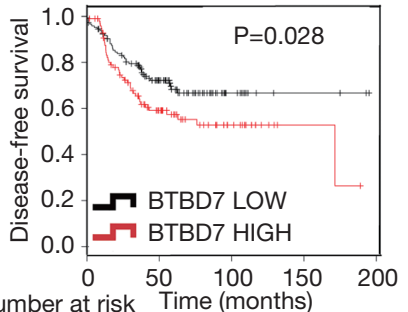

Number at risk Time (months)

Low $147 \quad 72 \quad 13 \quad 3 \quad 0$

High $99 \quad 41 \quad 13$

G

TNBC (IHC)

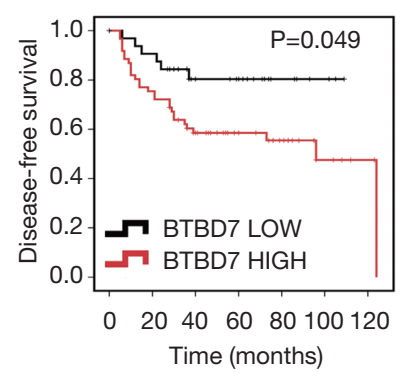

D Her-2 overexpression (TCGA)

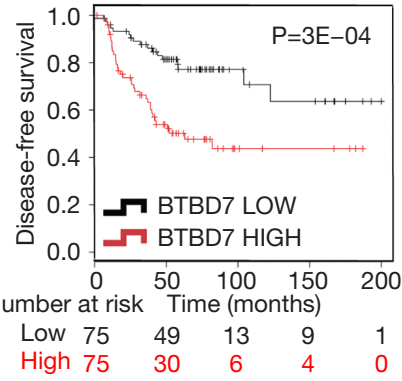

$\mathrm{H}$

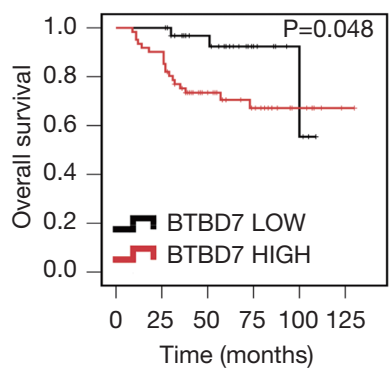

E

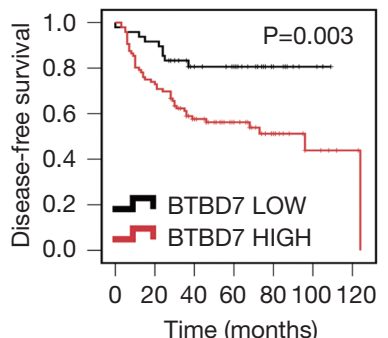

I Her-2 overexpression (IHC)

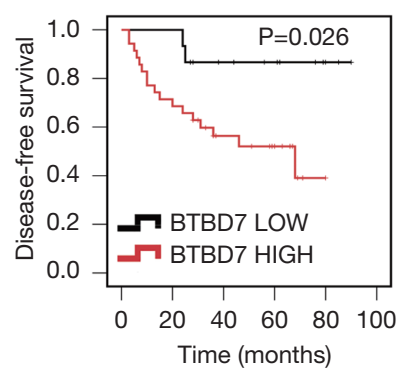

F $\quad \operatorname{HRNBC}(\mathrm{IHC})$

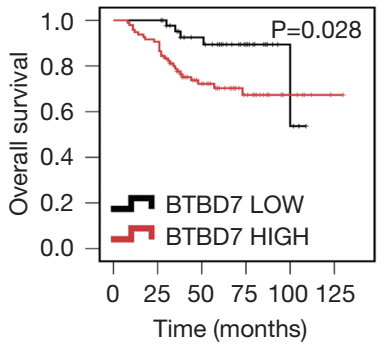

J Her-2 overexpression (IHC)

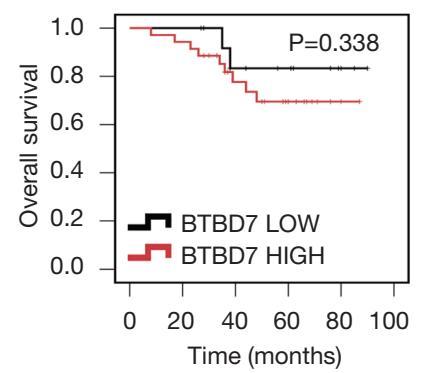

Figure 1 Expression level and prognostic value of BTBD7 in HRNBC. (A) Immunohistochemical staining of BTBD7 in benign breast lesions, NAT, and HRNBC tissues (400x); (B) Expression of BTBD7 differed by cancer or benign tissue, age, tumor volume, lymph node metastasis, TNM stage, and IDC differentiated degree (I-II for well to moderate, III for poor); (C,D) High BTBD7 mRNA level influences breast cancer prognosis in TCGA cohorts; (E-J) Kaplan-Meier curve analysis shows the DFS and OS in patients with HRNBC, TNBC, and HER-2 overexpression with high and low BTBD7 protein expression by immunohistochemical staining. NS, no significant difference, ${ }^{*} \mathrm{P}<0.05,{ }^{* *} \mathrm{P}<0.001$. HRNBC, hormone receptor-negative breast cancers; DFS, disease-free survival; OS, overall survival.

while no association could be detected between BTBD7 expression and bone, liver, brain metastasis, and chest wall recurrence $(\mathrm{P}>0.05)$ (Table 2).

\section{Correlation between BTBD7 and SLUG expression in HRNBC patients}

As SLUG is recognized to be an important regulatory factor in EMT, to verify the level of influence of BTBD7 on EMT, correlation analyses on BTBD7 and SLUG were performed. IHC (Figure 2A) and immunofluorescent (Figure 2B) staining in the HRNBC tumor tissue showed that SLUG protein was observed mainly in the cytoplasm and cell nucleus, and co-expression of BTBD7 and SLUG could be observed in tumor tissue.

TCGA data revealed that in HRNBC patients, those 
Table 2 BTBD7 expression level and metastatic and recurrence in HRNBC patients

\begin{tabular}{|c|c|c|c|c|}
\hline \multirow{2}{*}{$\begin{array}{l}\text { Metastatic and } \\
\text { recurrence sites }\end{array}$} & \multicolumn{2}{|c|}{ BTBD7 } & \multirow{2}{*}{$\begin{array}{l}\text { Correlation } \\
\text { coefficient }\end{array}$} & \multirow{2}{*}{$P$ value } \\
\hline & Low & High & & \\
\hline Lung & & & 0.282 & 0.001 \\
\hline- & 46 & 69 & & \\
\hline+ & 2 & 27 & & \\
\hline Bone & & & 0.102 & 0.223 \\
\hline- & 44 & 81 & & \\
\hline+ & 4 & 15 & & \\
\hline Liver & & & 0.019 & 0.817 \\
\hline- & 45 & 89 & & \\
\hline+ & 3 & 7 & & \\
\hline Chest wall & & & -0.031 & 0.708 \\
\hline- & 42 & 86 & & \\
\hline+ & 6 & 10 & & \\
\hline Brain & & & 0 & 1 \\
\hline- & 47 & 94 & & \\
\hline+ & 1 & 2 & & \\
\hline
\end{tabular}

-, no metastatic or recurrence; +, metastatic and recurrence in the corresponding organ. All metastatic or recurrence sites were confirmed by CT-scan or Pet-CT during the follow-up period. HRNBC, hormone receptor-negative breast cancers.

with high SLUG mRNA expression tended to have shorter DFS than those with low expression ( $\mathrm{P}=0.014$, Figure $2 C$ ), but there was no significant difference in OS between these groups (Figure 2D). Our IHC results also indicated that high SLUG protein expression in HRNBC patient tissue was associated with shorter DFS $(\mathrm{P}=0.001)$ and $\mathrm{OS}$ $(\mathrm{P}=0.003)$ (Figure $2 E, 2 F)$, which indicated that SLUG was also a prognostic indicator in HRNBC patients. The rate of high SLUG expression in patients with high BTBD7 was $72.9 \%(70 / 96)$, while the rate was $41.7 \%(20 / 48)$ in those with low BTBD7 (Table S1). A scatter diagram was performed to identify the correlation between these two markers, and the linear correlation coefficient was calculated to be 0.304 with a $\mathrm{P}$-value of less than 0.001 . As such, the expression of BTBD7 was weakly positively correlated with SLUG expression (Figure 2G).

Considering the prognostic significance of $\mathrm{BTBD} 7$ and SLUG, we generated receiver operating characteristic (ROC) curves to assess the predictive value of the 3- and
5 -year DFS rate. As shown in Figure 2H,2I, the area under the curve (AUC) in both BTBD7 and SLUG expression positive was 0.745 for 3 years and 0.735 for 5 years, which was higher than that in $\mathrm{pT}, \mathrm{pN}$, and BTBD7 or SLUG expression positive alone $(\mathrm{P}<0.05)$, which indicated that combination of SLUG and BTBD7 proteins expression levels had the better prognostic value in evaluating the HRNBC DFS rate.

\section{Univariate and multivariate analyses}

Furthermore, the factors potentially affecting HRNBC prognosis, including DFS (Table 3) and OS (Table 4), were analysed using a Cox regression model, and univariate Cox regression analysis showed that good prognostic factors for DFS were no lymphatic metastasis $(\mathrm{P}=0.038)$, lower TNM stage $(\mathrm{P}=0.007)$, well or moderate IDC differentiation $(\mathrm{P}=0.026)$, lower BTBD7 $(\mathrm{P}=0.003)$, and lower SLUG expression level $(\mathrm{P}=0.001)$. However, in multivariate $\mathrm{Cox}$ regression analysis, age $(\mathrm{P}=0.014)$, $\mathrm{BTBD} 7(\mathrm{P}=0.013)$, and SLUG $(\mathrm{P}=0.011)$ were independent prognostic factors. Univariate Cox regression analysis of the OS showed only younger age $(\mathrm{P}=0.006)$, lower $\mathrm{BTBD} 7$ expression levels $(\mathrm{P}=0.035)$, and lower SLUG expression levels $(\mathrm{P}=0.006)$ were good prognostic factors, while multivariate Cox regression analysis showed age $(\mathrm{P}=0.001)$, BTBD7 $(\mathrm{P}=0.036)$, and SLUG $(\mathrm{P}=0.021)$ were independent prognostic factors.

\section{BTBD7 promotes $M D A-M B-231$ cell metastasis via the EMT patbway}

As clinical data and IHC analyses demonstrated that high BTBD7 and SLUG were poor prognostic factors in HRNBC patients, and the EMT pathway may be the key element for understanding the molecular mechanism of BTBD7 and SLUG in breast cancer, it was important to identify the localization and function of these proteins within the cells.

MDA-MB-231 and SKBR-3 are cell lines with negative sex hormone receptors and had higher BTBD7 expression than immortalized mammary cells $(\mathrm{MCF}-10 \mathrm{~A})(\mathrm{P}<0.01$; Figure $3 A$ ). Immunofluorescence staining results (Figure $3 B$ ) demonstrated that MDA-MB-231 cells showed coexpression of SLUG and BTBD7 protein, which was consistent with the results obtained from HRNBC tissues.

Subsequently, we successfully knocked down the expression of BTBD7 using short interfering RNA in MDA-MB-231 cells, as shown in Figure 3C,3D, using 
A

Low expression

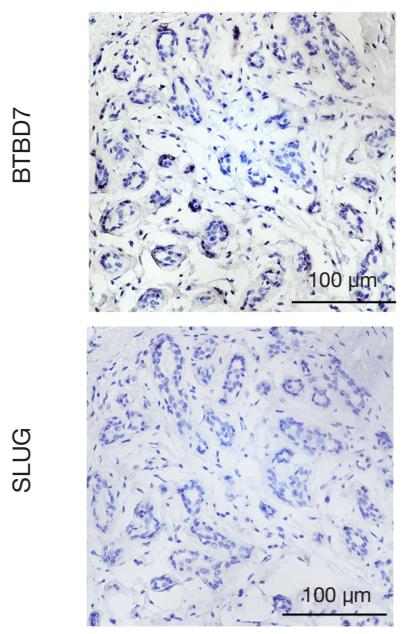

C

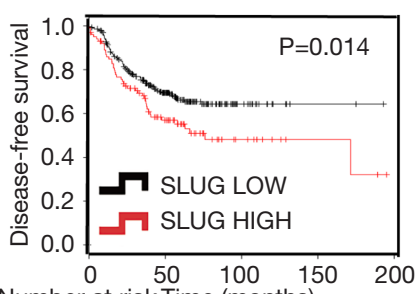

High expression

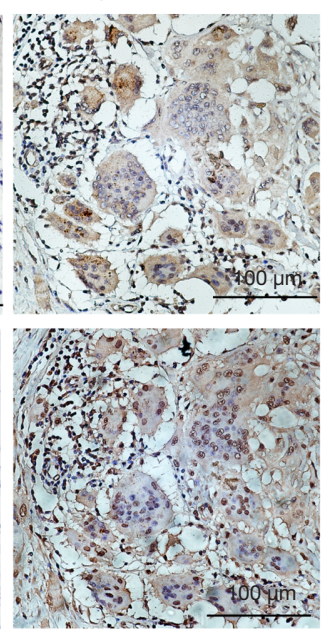

B

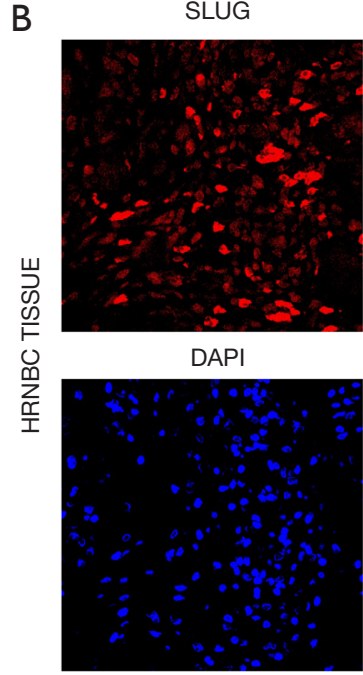

E

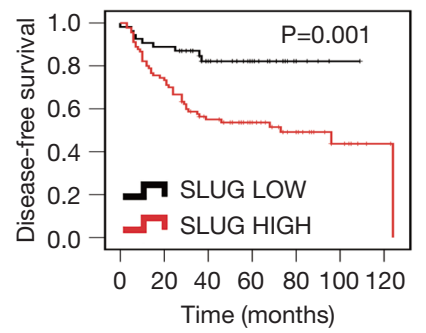

BTBD7

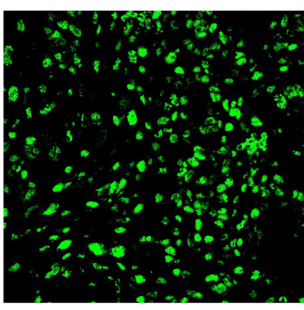

Merge

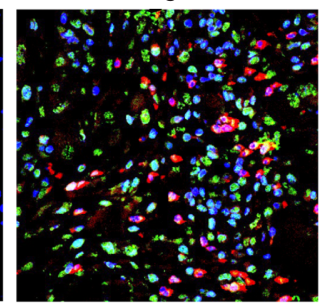

F HRNBC (SLUG IHC)

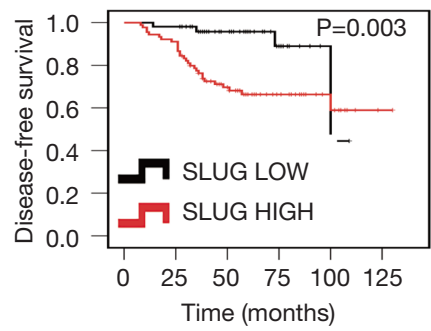

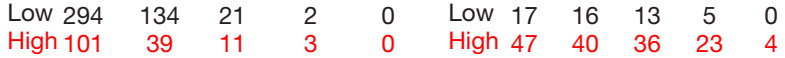

G

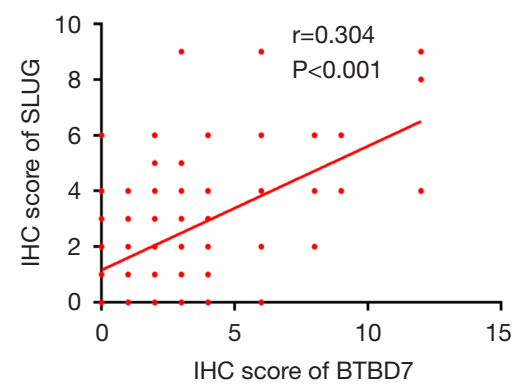

$\mathrm{H}$

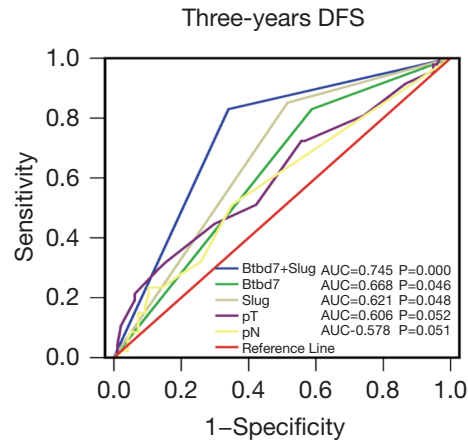

I

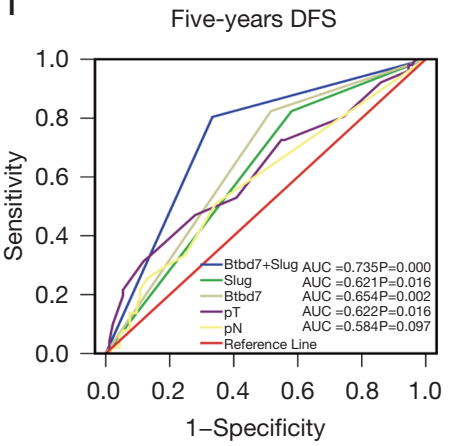

Figure 2 Immunohistochemical and immunofluorescence staining and correlation analysis of BTBD7 and SLUG. (A) Representative immunohistochemical staining showing co-expression of BTBD7 and SLUG in HRNBC tissues (400x); (B) representative immunofluorescence staining of co-expression of BTBD7 and SLUG in HRNBC tissues (400x); (C,D) high SLUG mRNA level influences prognosis in HRNBC patients in TCGA cohorts; (E,F) Kaplan-Meier curve analysis shows the DFS and OS in patients with HRNBC with high and low SLUG protein expression by immunohistochemical staining; $(\mathrm{G})$ linear regression of the expression level of BTBD7 and SLUG in HRNBC tissue by scatter diagram; (H,I) SLUG and BTBD7 predict 3- and 5-year DFS in HRNBC patients by ROC curves. DFS, disease-free survival; OS, overall survival; HRNBC, hormone receptor-negative breast cancers. 
Table 3 RFS of HRNBC patients based on univariate and multivariate Cox proportional regression analyses.

\begin{tabular}{|c|c|c|c|c|}
\hline Factor & \multicolumn{2}{|c|}{ Univariate } & \multicolumn{2}{|c|}{ Multivariate } \\
\hline Age ( $<50$ vs. $\geq 50$ years) & $1.71(0.99-2.97)$ & 0.056 & $2.05(1.16-3.64)$ & 0.014 \\
\hline Tumor diameter $(<2$ vs. $\geq 2 \mathrm{~cm})$ & $1.47(0.72-3.02)$ & 0.291 & - & - \\
\hline Lymphatic metastasis (yes vs. no) & $1.78(1.03-3.08)$ & 0.038 & - & - \\
\hline Her-2 (- vs. +) & $1.20(0.67-2.10)$ & 0.549 & - & - \\
\hline IDC grading (I-II vs. III) & $2.00(1.09-3.68)$ & 0.026 & - & - \\
\hline BTBD7 (low vs. high) & $3.13(1.47-6.64)$ & 0.003 & $2.70(1.23-5.91)$ & 0.013 \\
\hline SLUG (low vs. high) & $3.66(1.72-7.78)$ & 0.001 & $2.75(1.27-6.00)$ & 0.011 \\
\hline
\end{tabular}

HRNBC, hormone receptor-negative breast cancers.

Table 4 OS of HRNBC patients based on univariate and multivariate Cox proportional regression analyses

\begin{tabular}{lccc}
\hline \multirow{2}{*}{ Factor } & \multicolumn{1}{c}{ Univariate } & & Multivariate \\
\cline { 2 - 4 } & $\mathrm{HR}(95 \% \mathrm{Cl})$ & $\mathrm{P}$ value & $\mathrm{HR}(95 \% \mathrm{Cl})$ \\
\hline Age $(<50$ vs. $\geq 50$ years) & $1.71(0.99-2.97)$ & 0.056 & $2.05(1.16-3.64)$ \\
Tumor diameter (<2 vs. $\geq 2$ cm) & $1.47(0.72-3.02)$ & 0.291 & - \\
Lymphatic metastasis (yes vs. no) & $1.78(1.03-3.08)$ & 0.038 & - \\
TNM (I-II vs. III-IV) & $2.20(1.24-3.98)$ & 0.007 & - \\
Her-2 (- vs. +) & $1.20(0.67-2.10)$ & 0.549 & - \\
IDC grading (I-II vs. III) & $2.00(1.09-3.68)$ & 0.026 & - \\
BTBD7 (low vs. high) & $3.13(1.47-6.64)$ & 0.003 & - \\
SLUG (low vs. high) & $3.66(1.72-7.78)$ & 0.001 & $2.70(1.23-5.91)$ \\
\hline
\end{tabular}

OS, overall survival; HRNBC, hormone receptor-negative breast cancers.

qRT-PCR and western blot analysis. While BTBD7 expression was silenced in MDA-MB-231 cells, the epithelial marker E-cadherin was up-regulated $(\mathrm{P}<0.05)$, and the mesenchymal markers $\alpha$-smooth muscle actin protein $(\alpha-S M A)$ and SLUG $(\mathrm{P}<0.05)$ were down-regulated, indicating that the EMT process was inhibited (Figure $3 D$ ). To corroborate the function of BTBD7 in MDA-MB-231 cells, cell invasion assays were used to analyse cell invasion ability and showed lower invasive properties in BTBD7siRNA groups than in the control group (Figure $3 E$ ).

\section{BTBD7 is associated with generic transcription of breast cancer}

To explore the functional roles of $B T B D 7$ in breast cancer, we screened out 332 DEGs (in total online: https://cdn. amegroups.cn/static/public/atm-21-3409-1.xlsx) according to the expression level of $B T B D 7$. The top three upregulated genes were TRIP11, DICER1, and $A T G 2 B$, and the top three down-regulated genes were LSM4, PAM16, and SNRPA. DAVID was used to analyse the DEGs and enriched 30 GO terms (Figure 4A, Table S2), including DNA-templated transcription, regulation of transcription, and integral component of the membrane. To enlarge this observation, KOBAS 3.0 was performed, and 25 KEGG terms (Figure 4B, Table S3) including signal transduction and generic transcription pathways, were obtained. These results revealed that $B T B D 7$ may be associated with generic transcription, which may be the mechanism by which it promotes tumor invasion and metastasis. 
A

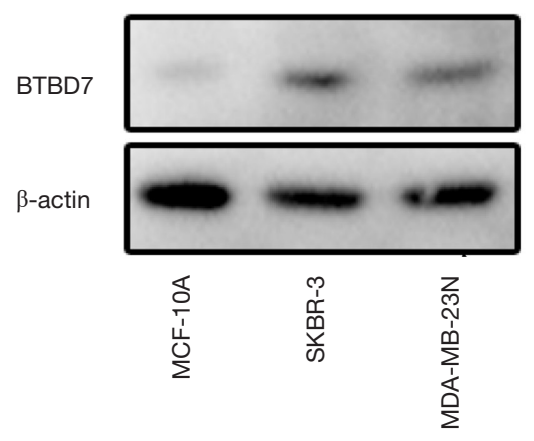

D

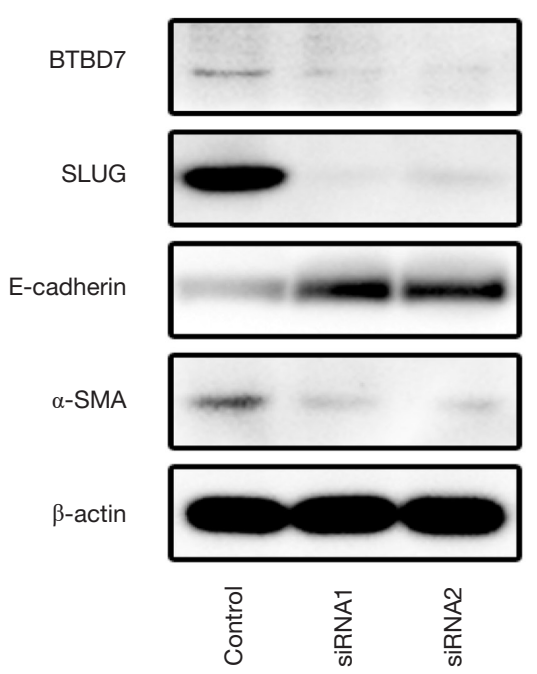

B

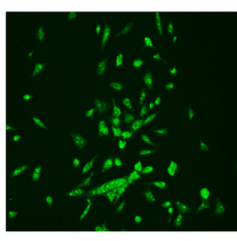

DAPI
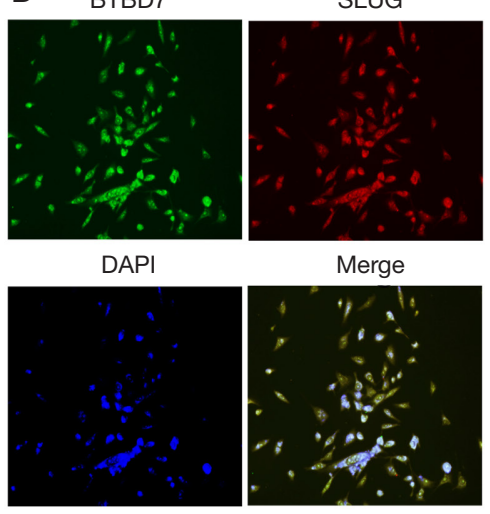

C

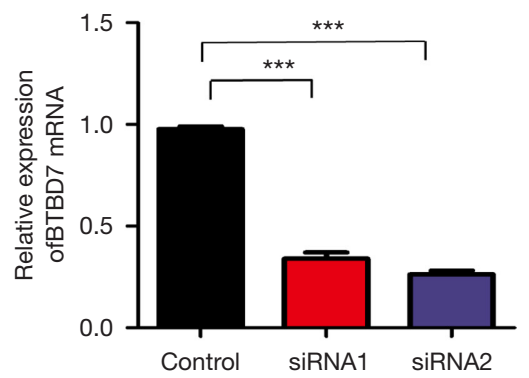

E
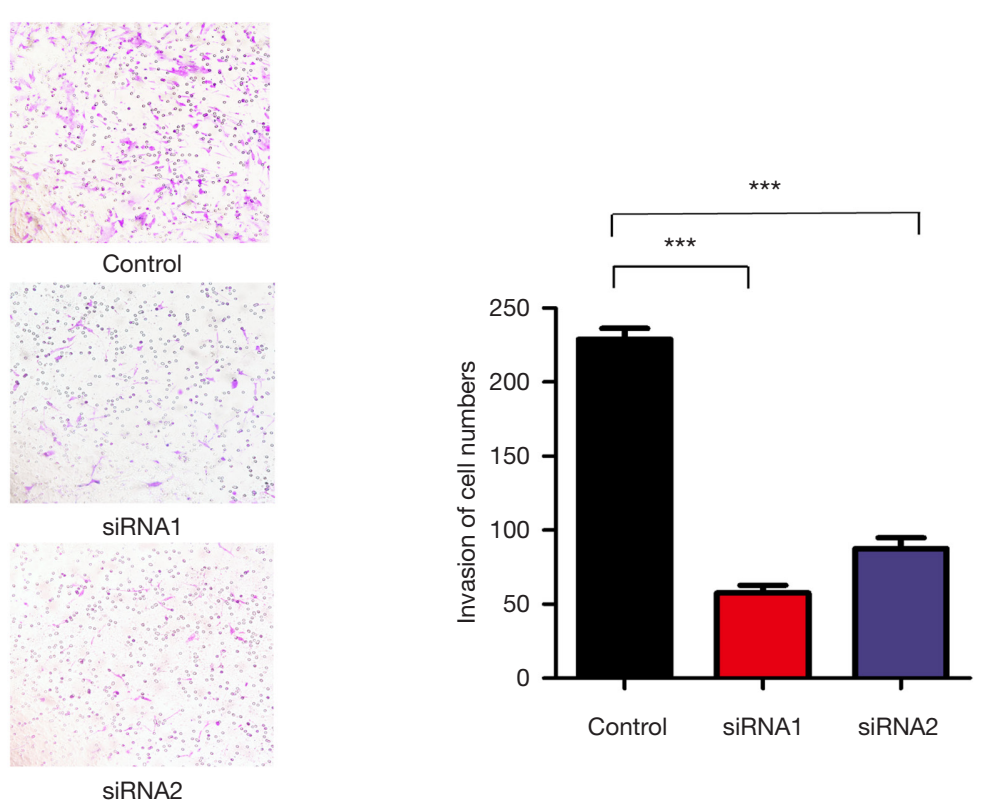

Figure 3 Localization and function of BTBD7 in HRNBC cells. (A) Western blot analysis results for BTBD7 protein in MCF-10A, SKBR-3, and MDA-MB-231 cells; (B) immunohistochemical staining of co-expression of BTBD7 and SLUG in MDA-MB-231 cells (400x); (C) RTPCR analysis results of the BTBD7 mRNA expression in the MDA-MB-231 cells treated with BTBD7 siRNAs and in the control group; (D) Western blot analysis results for BTBD7, SLUG, E-cadherin, and $\alpha$-SMA expression in the MDA-MB-231 cells treated with BTBD7 siRNAs and in the control group; (E) results of cell migration assay in MDA-MB-231 cells treated with BTBD7 siRNAs and in the control group (200×). ${ }^{* * *} \mathrm{P}<0.001$. HRNBC, hormone receptor-negative breast cancers.

\section{Discussion}

Cancer metastasis is the leading causes of death in patients with malignant tumors (26-29). Our previous research (30) has shown that different BRCA molecular subtypes had significantly different prognoses. Traditional prognostic indicators such as TNM staging and pathological grade was the factors affecting the prognosis of hormone receptor- positive breast cancer. However, the factors affecting in HRNBC were more complicated, our study aimed to determine the predictive factors for HRNBC.

In immunohistochemical and different cell lines studies, BTBD7 protein was mainly expressed in the cytoplasm of tumor cells, with significantly higher levels in HRNBC tumor tissue and in negative sex hormone receptor breast 
A

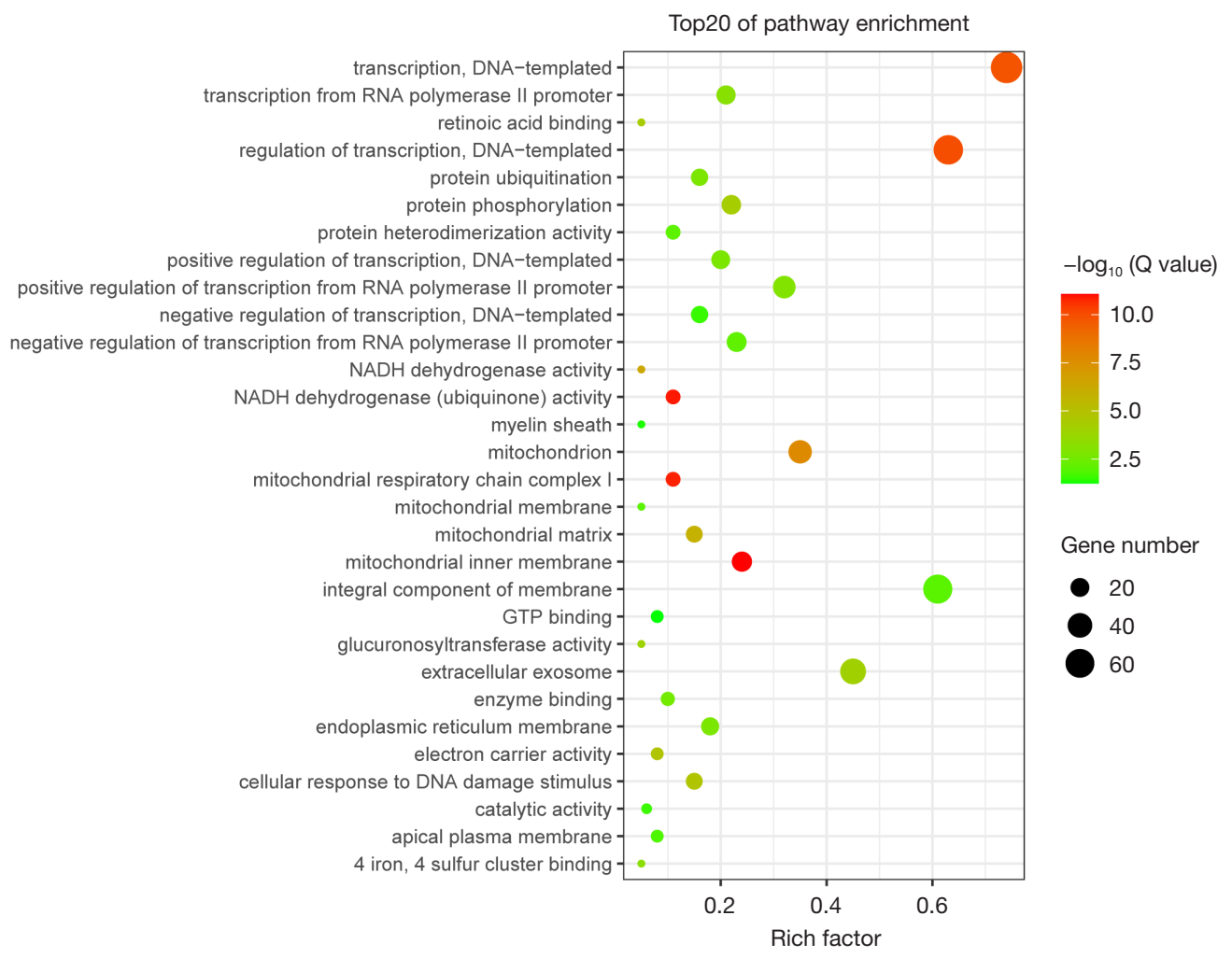

B

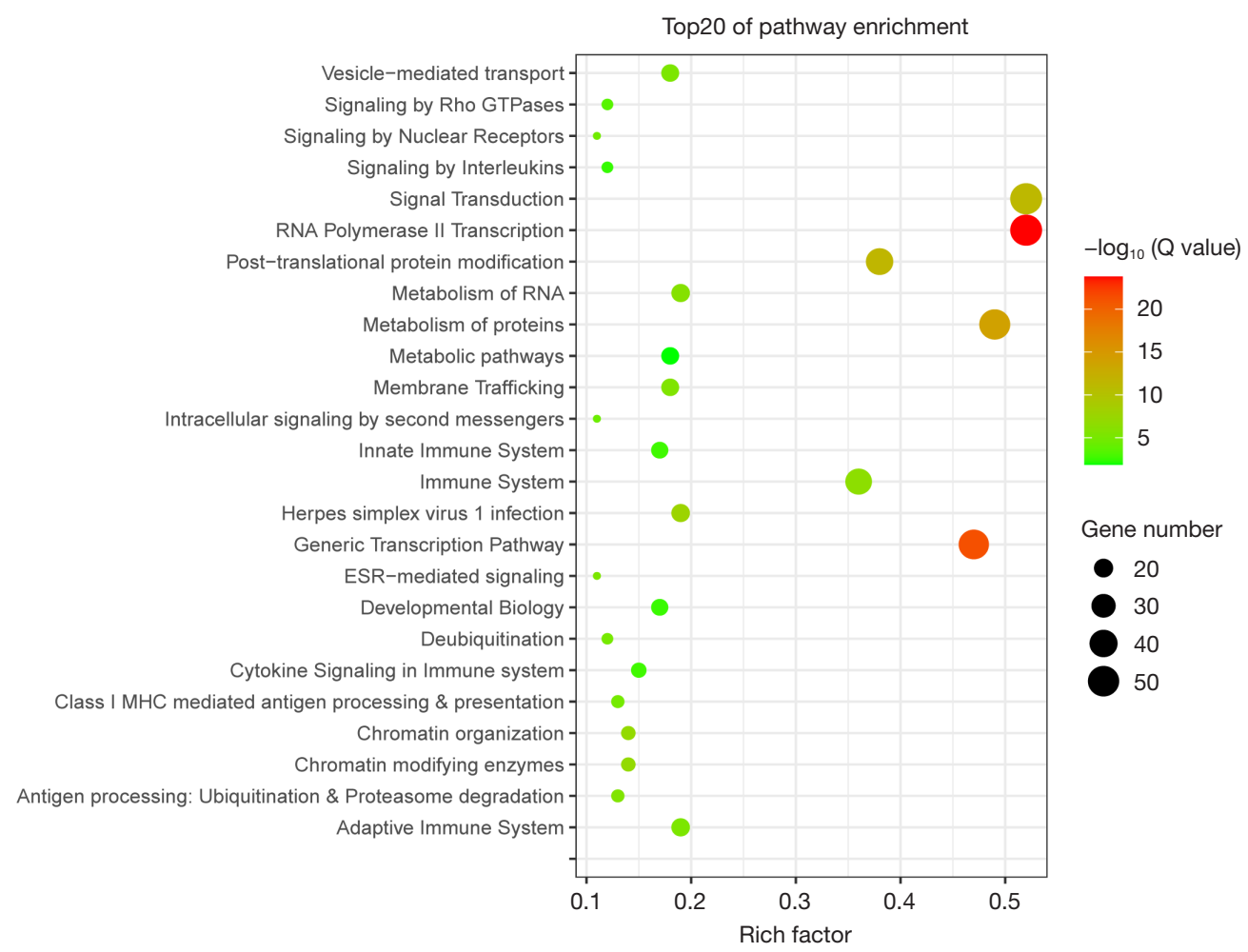

Figure 4 Bioinformatics analysis of BTBD7 gene function in breast cancer. (A) DAVID was used to analyse the 332 DEGs and enriched 30 GO terms, such as DNA-templated transcription, regulation of transcription, and integral component of the membrane; (B) KOBAS 3.0 was performed and 25 KEGG terms such as signal transduction and generic transcription pathway were obtained. 
cancer cells than in the NAT, benign lesion tissue, and immortalized mammary cells. Our results were similar to those derived from Fan et al. (31), which showed BTBD7 expression was elevated in NSCLC tissues compared with normal lung tissues. This suggests that BTBD7 is a probable biomarker for malignant cancer. Fan et al. (31) further observed that increased BTBD7 expression in NSCLC was significantly associated with lymph node metastasis and advanced TNM stages. Liu et al. (32) found that in salivary adenoid cystic carcinoma, positive rates of BTBD7 expression were significantly associated with lymph node metastasis. In our study, the pathological analysis showed that the overexpression of BTBD7 in HRNBC was associated with larger tumor volume as well as with poorer TNM stages. This suggests BTBD7 may be an important molecule promoting the malignant behaviour of tumors.

TCGA database and immunohistochemical analysis demonstrated that HRNBC patients with high BTBD7 mRNA expression levels had a poor prognosis. A study conducted by Tao et al. (21) showed that BTBD7 mRNA expression in hepatocarcinoma could promote cancer cell proliferation. In addition, Luo et al. (20) observed that NSCLC patients with negative BTBD7 expression had a longer OS time than those with positive expression.

TNBC subgroup analyses indicated that in patients aged $<50$ years and TNM stages $1-2$, with well + moderately differentiated invasive ductal carcinoma, high $B T B D 7$ expression indicated a shorter DFS, and in patients age $>50$ years and with lymph node metastasis, high BTBD7 expression indicated a shorter OS. Subgroup analysis of patients with HER-2 overexpression indicated that among patients aged $>50$ years, tumor diameter $>2 \mathrm{~cm}$, lymph node metastasis, poorly differentiated IDC, and a higher BTBD7 expression level were associated with shorter DFS time. However, we did not find any significant association with OS among HER-2 + patients, indicating a larger sample size is required, and suggests clinical doctors should pay more attention to this patient population who exhibit BTBD7 expression.

A study by Onodera et al. (19) showed that BTBD7 was a dynamic regulator of branching morphogenesis and was required for the branching of embryonic mammalian salivary glands and lungs. As described in previous studies (20,31), BTBD7 may contribute to lung cancer development and poor clinical outcome in patients with NSCLC. Our study has further proved that high BTBD7 expression can contribute to the development of metastatic lung cancer $(\mathrm{P}=0.001)$, and as a result, we suggest that annual chest radiography or chest computed tomography examination should be performed in HRNBC patients with a high level of BTBD7 expression.

SLUG is an important transcriptional factor regulating the expression of genes responsible for the EMT $(16,33)$, and has been demonstrated to downregulate epithelial markers such as E-cadherin, as well as the up-regulate the expression of mesenchymal markers such as $\mathrm{N}$-cadherin and fibronectin. Moreover, Yang et al. $(22,34)$ identified a positive correlation between BTBD7 and SLUG expression in SACC tissues, and BTBD7 silencing inhibited the expression of SLUG in SACC cells. TCGA data and IHC experiments in our study illustrated a common result showing that at both mRNA and protein level, a high SLUG expression was associated with poor prognosis in HRNBC. Immunohistochemical and immunofluorescent staining of HRNBC tumor tissue and MDA-MB-231 cells verified the co-expression of BTBD7 and SLUG in cells, and the ROC curves of the 3- and 5-year DFS showed that combining BTBD7 and SLUG expressions had the best predictive value for HRNBC recurrence.

Multivariatee Cox regression analyses revealed that AGE, BTBD7, and SLUG were independent prognostic factors for DFS and OS, and in AGE, BTBD7, and SLUG, a low value was associated with better outcomes. Although the univariate Cox regression analyses showed lymphatic metastasis, TNM, and IDC grading were the prognostic factors for DFS, which has been widely accepted clinically, we found that BTBD7 and SLUG were better prognostic factors, which may indicate that BTBD7 and SLUG are more sensitive in forecasting HRNBC prognosis.

As mentioned above, BTBD7 may play a significant role in EMT in HRNBC, and to confirm this conjecture, we knocked down BTBD7 in MDA-MB-231 cells.

After the BTBD7 expression was silenced, the SLUG protein was significantly down-regulated, and as a result, the EMT process was inhibited. These findings suggest that $B T B D 7$ positively regulates the EMT process, which is consistent with those of other reports $(21,22,32)$. In addition, invasion assays identified that $B T B D 7$ can promote the invasion ability of the cells, which was in accordance with the IHC results.

To further explore the mechanism by which the BTBD7 gene plays a role in breast cancer, we screened out 332 DEGs according to the expression level of BTBD7 by bioinformatics analysis. As more research is required to elucidate the relationship between the altered expression of these genes and BTBD7. Following GO and KEGG 
analyses, it was identified that $B T B D 7$ may be associated with the generic transcription, integral component of the membrane, and signal transduction. Tian et al. (17) showed that the promoter region of the $\mathrm{BTB} / \mathrm{POZ}$ domain contains binding sites for notable transcription factors such as alphafetoprotein (AFP)-1 and GATA in HepG2 cells, which was consistent with our results. However, further in vitro investigations are required to fully elucidate the mechanism of BTBD7 action in cancer cells.

In summary, this study reports that high levels of BTBD7 and SLUG expression were significantly related to metastasis OS in HRNBC patients and were poor prognostic factors. We further identified the co-expression of BTBD7 and SLUG in HRNBC tissues and cells and found BTBD7 was an important contributor to EMT regulation. Moreover, BTBD7 may be associated with generic transcription, which may influence proliferation and metastasis in breast cancer. BTBD7 might act as a potential molecular target for gene therapy in HRNBC patients.

\section{Acknowledgments}

Funding: This work was supported by National Natural Science Foundation of China (No. 81402509 to TTW, No. 81672640 to LL), Department of Education of Guangdong Province (2020KQNCX021 to ZXL), the Grant for Key Disciplinary Project of Clinical Medicine under the Guangdong High-level University Development Program, the Project of Innovating and Strengthening Universities in Guangdong Province (No. 2018KTSCX066 to LL), the Special Funds for Innovation Strategy of Science and Education in Guangdong Province (No. 2018-157 to LL), Key Project of Natural Science Foundation of Bengbu Medical College (BYKY1826ZD to XKM), and Key Projects of Natural Science Foundation of Anhui Province University (KJ2019A0347 to XKM).

\section{Footnote}

Reporting Checklist: The authors have completed the REMARK reporting checklist. Available at https://dx.doi. org/10.21037/atm-21-3409

Data Sharing Statement: Available at https://dx.doi. org/10.21037/atm-21-3409

Conflicts of Interest: All authors have completed the ICMJE uniform disclosure form (available at https://dx.doi. org/10.21037/atm-21-3409). The authors have no conflicts of interest to declare.

Ethical Statement: The authors are accountable for all aspects of the work in ensuring that questions related to the accuracy or integrity of any part of the work are appropriately investigated and resolved. Ethics approval was obtained from the ethics committee of The Third Affiliated Hospital of Sun Yat-sen University (No. [2018]-081) and the First Affiliated Hospital, Shantou University Medical College (No. [2018]-027). Samples were anonymously coded in accordance with local ethical guidelines [as stipulated by the Declaration of Helsinki (as revised in 2013)]. Written informed consent was obtained from study participants.

Open Access Statement: This is an Open Access article distributed in accordance with the Creative Commons Attribution-NonCommercial-NoDerivs 4.0 International License (CC BY-NC-ND 4.0), which permits the noncommercial replication and distribution of the article with the strict proviso that no changes or edits are made and the original work is properly cited (including links to both the formal publication through the relevant DOI and the license). See: https://creativecommons.org/licenses/by-nc-nd/4.0/.

\section{References}

1. Bray F, Ferlay J, Soerjomataram I, et al. Global cancer statistics 2018: GLOBOCAN estimates of incidence and mortality worldwide for 36 cancers in 185 countries. CA Cancer J Clin 2018;68:394-424.

2. Li T, Mello-Thoms C, Brennan PC. Descriptive epidemiology of breast cancer in China: incidence, mortality, survival and prevalence. Breast Cancer Res Treat 2016;159:395-406.

3. Siegel RL, Miller KD, Jemal A. Cancer statistics, 2018. CA Cancer J Clin 2018;68:7-30.

4. Kohler BA, Sherman RL, Howlader N, et al. Annual Report to the Nation on the Status of Cancer, 19752011, Featuring Incidence of Breast Cancer Subtypes by Race/Ethnicity, Poverty, and State. J Natl Cancer Inst 2015;107:djv048. Erratum in: J Natl Cancer Inst 2015;107:djv121.

5. Ross JS, Slodkowska EA, Symmans WF, et al. The HER2 receptor and breast cancer: ten years of targeted antiHER-2 therapy and personalized medicine. Oncologist 2009;14:320-68. 
6. Wei W, Cao S, Liu J, et al. Fibroblast growth factor receptor 4 as a prognostic indicator in triple-negative breast cancer. Transl Cancer Res 2020;9:6881-8.

7. Carey L, Winer E, Viale G, et al. Triple-negative breast cancer: disease entity or title of convenience? Nat Rev Clin Oncol 2010;7:683-92.

8. Oikawa M. The history, present situation, and future directions of neoadjuvant chemotherapy for HER2negative breast cancer. Chin Clin Oncol 2020;9:29.

9. Noh JM, Choi DH, Huh SJ, et al. Patterns of recurrence after breast-conserving treatment for early stage breast cancer by molecular subtype. J Breast Cancer 2011;14:46-51.

10. De Laurentiis M, Cianniello D, Caputo R, et al. Treatment of triple negative breast cancer (TNBC): current options and future perspectives. Cancer Treat Rev 2010;36 Suppl 3:S80-6.

11. Zhao S, Zuo WJ, Shao ZM, et al. Molecular subtypes and precision treatment of triple-negative breast cancer. Ann Transl Med 2020;8:499.

12. Das V, Bhattacharya S, Chikkaputtaiah C, et al. The basics of epithelial-mesenchymal transition (EMT): A study from a structure, dynamics, and functional perspective. J Cell Physiol 2019. [Epub ahead of print]. doi:10.1002/ jcp.28160.

13. Kalluri R, Weinberg RA. The basics of epithelialmesenchymal transition. J Clin Invest 2009;119:1420-8.

14. Hajra KM, Chen DY, Fearon ER. The SLUG zinc-finger protein represses E-cadherin in breast cancer. Cancer Res 2002;62:1613-8.

15. Xin L, Zhao R, Lei J, et al. SND1 acts upstream of SLUG to regulate the epithelial-mesenchymal transition (EMT) in SKOV3 cells. FASEB J 2019;33:3795-806.

16. Zhang W, Sun J, Chen J, et al. Downregulation of miR95 in gastric cancer promotes EMT via regulation of Slug, thereby promoting migration and invasion. Oncol Rep 2019;41:1395-403.

17. Tian JM, Schibler U. Tissue-specific expression of the gene encoding hepatocyte nuclear factor 1 may involve hepatocyte nuclear factor 4. Genes Dev 1991;5:2225-34.

18. Daley WP, Matsumoto K, Doyle AD, et al. Btbd7 is essential for region-specific epithelial cell dynamics and branching morphogenesis in vivo. Development 2017;144:2200-11.

19. Onodera T, Sakai T, Hsu JC, et al. Btbd7 regulates epithelial cell dynamics and branching morphogenesis. Science 2010;329:562-5.

20. Luo FY, Liu ZH, Hu QH, et al. Association of BTBD7 with Metastasis and Poor Prognosis in Non-Small-Cell Lung Cancer Patients. J Cancer 2015;6:477-81.

21. Tao YM, Huang JL, Zeng S, et al. BTB/POZ domaincontaining protein 7: epithelial-mesenchymal transition promoter and prognostic biomarker of hepatocellular carcinoma. Hepatology 2013;57:2326-37.

22. Yang L, Wang T, Zhang J, et al. BTBD7 silencing inhibited epithelial- mesenchymal transition (EMT) via regulating Slug expression in human salivary adenoid cystic carcinoma. Cancer Biomark 2017;20:461-8.

23. Györffy B, Lanczky A, Eklund AC, et al. An online survival analysis tool to rapidly assess the effect of 22,277 genes on breast cancer prognosis using microarray data of 1,809 patients. Breast Cancer Res Treat 2010;123:725-31.

24. Huang da W, Sherman BT, Lempicki RA. Systematic and integrative analysis of large gene lists using DAVID bioinformatics resources. Nat Protoc 2009;4:44-57.

25. Xie C, Mao X, Huang J, et al. KOBAS 2.0: a web server for annotation and identification of enriched pathways and diseases. Nucleic Acids Res 2011;39:W316-22.

26. Polcz MM, Sebaratnam DF, Fernández-Peñas P. Atypical fibroxanthoma management: Recurrence, metastasis and disease-specific death. Australas J Dermatol 2018;59:10-25.

27. Nystrom LM. CORR Insights ${ }^{\circledR}$ : Can Multistate Modeling of Local Recurrence, Distant Metastasis, and Death Improve the Prediction of Outcome in Patients With Soft Tissue Sarcomas? Clin Orthop Relat Res 2017;475:1436-8.

28. Jung SW, Kim DS, Yu YD, et al. Risk factors for cancer recurrence or death within 6 months after liver resection in patients with colorectal cancer liver metastasis. Ann Surg Treat Res 2016;90:257-64.

29. Thompson AK, Kelley BF, Prokop LJ, et al. Risk Factors for Cutaneous Squamous Cell Carcinoma Recurrence, Metastasis, and Disease-Specific Death: A Systematic Review and Meta-analysis. JAMA Dermatol 2016;152:419-28.

30. Zenan H, Zixiong L, Zhicheng Y, et al. Clinical prognostic evaluation of immunocytes in different molecular subtypes of breast cancer. J Cell Physiol 2019;234:20584-602.

31. Fan C, Miao Y, Zhang X, et al. Btbd7 contributes to reduced $\mathrm{E}$-cadherin expression and predicts poor prognosis in non-small cell lung cancer. BMC Cancer. 2014;14:704. Published 2014 Sep 24. doi:10.1186/1471-2407-14-704

32. Liu Y, Song J, Zhang J, et al. BTB/POZ domaincontaining protein 7 is inversely associated with fibronectin expression in salivary adenoid cystic carcinoma. Oral Surg Oral Med Oral Pathol Oral Radiol 2018;125:468-77.

33. Krstic M, Kolendowski B, Cecchini MJ, et al. TBX3 
promotes progression of pre-invasive breast cancer cells by inducing EMT and directly up-regulating SLUG. J Pathol 2019;248:191-203.

Cite this article as: $\mathrm{Li} \mathrm{ZX}$, Huang ZN, Luo H, Yang XB, Wang YL, Chen JX, Ma XK, Xu F, Wang TT, Lin L. High BTBD7 expression positive is correlated with SLUG-predicted poor prognosis in hormone receptor-negative breast cancer. Ann Transl Med 2021;9(15):1252. doi: 10.21037/atm-21-3409
34. Yang L, Wang T, Zhang J, et al. Expression of BTBD7 in primary salivary adenoid cystic carcinoma and correlation with Slug and prognosis. Cancer Biomark 2016;17:179-85. 